\title{
La politique de la Terreur. Essai sur la violence révolutionnaire (1789-1794).
}

\section{Claude Mazauric}

\section{(2) OpenEdition}

1 Journals

\section{Édition électronique}

URL : https://journals.openedition.org/ahrf/1026

DOI : 10.4000/ahrf.1026

ISSN : 1952-403X

Éditeur :

Armand Colin, Société des études robespierristes

\section{Édition imprimée}

Date de publication : 1 mars 2001

Pagination : 118-121

ISSN : 0003-4436

\section{Référence électronique}

Claude Mazauric, "La politique de la Terreur. Essai sur la violence révolutionnaire (1789-1794). »,

Annales historiques de la Révolution française [En ligne], 323 I janvier-mars 2001, mis en ligne le 21 avril 2004, consulté le 24 avril 2022. URL : http://journals.openedition.org/ahrf/1026 ; DOI : https://doi.org/ 10.4000/ahrf.1026

Ce document a été généré automatiquement le 24 avril 2022.

Tous droits réservés 


\title{
La politique de la Terreur. Essai sur la violence révolutionnaire
} (1789-1794).

\author{
Claude Mazauric
}

\section{RÉFÉRENCE}

Patrice Gueniffey, La politique de la Terreur. Essai sur la violence révolutionnaire (1789-1794), Paris, Fayard, 2000, 376 p.

1 L'ouvrage de Patrice Gueniffey veut combler une lacune car selon l'auteur «on n'écrit plus guère sur la Terreur» (p.1). Cette affirmation par laquelle commence le livre, surprendra assurément ceux qui ont en mémoire (et en fiches!) les innombrables travaux, recherches, études, colloques, qui ont relancé depuis vingt ans, en France et dans nombre de pays, le débat sur la Terreur, son économie, ses effets et son substrat historique, psychologique, idéologique, etc. Ne retenons en ce domaine que le Colloque de Stanford (1992, actes parus en 1994) que patronnèrent Keith Baker et Colin Lucas, colloque finalement pluraliste et attentif à rendre compte des points de vue divergents, colloque auquel ici ou là se réfère Gueniffey mais en ne retenant du lot que ce qui s'y est dit à son avantage. Sans doute faut-il considérer plus généralement que nous ne lisons pas les mêmes textes, ni ne fréquentons les mêmes bibliothèques ou librairies que M.Gueniffey! N'accordons cependant pas à cette affirmation péremptoire plus d'importance qu'il n'en faut: c'est après tout la liberté de l'essayiste que de tenir pour fatras érudit, guenilles idéologiques ou billevisées d'illusionnistes, ce que d'autres que lui-même et ceux auxquels il accorde crédit, ont pu se donner la peine d'énoncer avant lui, et ne retenons que la démarche qui inspire l'essai et la qualité de la matière qui constitue son ouvrage, un ouvrage se voulant d'ailleurs moins une histoire de la Terreur révolutionnaire de 1789 à 1794 (exclusivement) qu'une «réflexion sur la politique de la Terreur et la violence révolutionnaire» (p.12). 
2 Conduite comme une série de conférences dont l'origine serait un enseignement dispensé à l'Université de Macerata en 1997, la mise en œuvre de l'essai de Gueniffey ne manque pas d'inscrire son aboutissement sous l'amical patronage de quelques éminents directeurs d'études de l'E.H.E.S.S. (Paris) - mais pas de tous, on le remarquera aisément!- et dans une référence appuyée à l'œuvre de François Furet dont l'influence posthume se lit tout au long du livre. En onze chapitres qui valent chacun comme une manière d'essai plus ou moins autonome, relatif à un aspect particulier du rapport de la politique à la Terreur et à la violence révolutionnaire, se construit une démarche d'ensemble dont la clarté et la finalité n'apparaissent pas toujours clairement, au-delà de la thèse initiale qui l'inspire. Ainsi aura-t-on un chapitre sur «Les circonstances et la guerre» (V), sur «Le moment de Prairial» (X), sur «Le jacobinisme» (VIII), ou au début sur la vision populaire de la souveraineté (III, «La leçon de l'Ami du peuple») qui énoncent des points de vue ordinairement exposés ailleurs et bien connus, à côté de propositions plus originales et neuves, comme dans le chapitre II («La Révolution française et la Terreur») qui a valeur d'un essai comparatif des expériences révolutionnaires antérieures et prêtant à de sérieuses discussions, ou dans le chapitre VII («Le Contrat social à l'épreuve de l'exception») qui oblige à revenir par le détour de la théorie politique, sur la configuration des champs idéologiques, des pratiques politiques et des héritages multiples, contribuant à faire du moment de la Terreur, dans la limitation chronologique retenue, un phénomène d'une plus vaste ampleur que sa réduction à l'exceptionnel comme on le pensait autrefois.

3 La thèse fondamentale de l'essai de Gueniffey, celle qui parcourt tout le livre, se résume en une proposition assez élémentaire: la Terreur n'a pas constitué un moment particulier et singulier de la Révolution, elle fut simplement la mise en forme, d'ailleurs morphologiquement changeante et institutionnellement différentielle, d'une violence organique consubstantielle à la Révolution elle-même, en tant qu'elle fut acte en fin de compte démiurgique. Cela n'a pas signifié comme on pourrait le croire que 1789 portait fatalement en soi la violence terroriste postérieure, car «la transformation du réel par l'action de la volonté n'est pas en elle-même un phénomène nécessairement terroriste» (p.50), sinon quel pourrait être le statut de la politique? Mais «la Terreur est un aboutissement plausible, voire dans certains cas fatal, lorsque l'action politique méconnaissant les limites que lui opposent les circonstances, prétend atteindre toutes les fins qu'elle s'est assignées». L'énonciation à front inversé de cette inattendue «théorie des circonstances» conduit à affirmer que la volonté transformatrice devient terroriste quand elle se fait «absolue», renonciation au «compromis» entre «bien rationnellement conçu» et «pesanteurs réelles»: une profession de foi tout benoîtement réformiste, candidement énoncée, qui pourrait constituer à tout prendre le fil conducteur de toutes les analyses contenues dans l'essai, un essai, je le signale en passant, qui rejette comme d'une guigne, toute référence positive à «l'histoire culturelle», tenue en fin de compte comme une manière de justification de la Terreur par l'attention qu'elle invite à porter sur l'exploration des pratiques, habitus et mentalités: on voit bien ici que c'est l'œuvre de Michel Vovelle qui est évacuée. Cependant, comme toute action politique, serait-elle radicale ou pas, révolutionnaire ou non, finit toujours par un compromis entre l'objectif recherché et les résistances opposées par les durs pépins de la réalité, en produire le constat, même en lui donnant la qualité d'une norme a priori souhaitable, ne nous éclaire guère comme historiens, sur la nature des processus, la nécessité ou la dynamique des événements ou des politiques qui pour changer peu de choses - ... et la Révolution en est modifiée beaucoup en 
profondeur! - ont été conduits plus ou moins volontairement à en bousculer beaucoup et bien au-delà du souhaitable ou du prévisible de manière à en consolider quelques-unes, pas forcément d'ailleurs dans ce qui à l'origine paraissait le plus nécessaire à réformer. Mais de cela qui renvoie aux enjeux de la Révolution, il n'est à peu près jamais question dans le livre de Gueniffey et, s'il cite à l'occasion Jaurès ( $c f$. $p$. 133, p.164, p. 245, pp. 273-274), lequel (re)devient curieusement avec ce livre et dans ce champ historiographique, une référence obligée après un quart de siècle d'occultation de sa démarche essentielle quand d'autres, comme Soboul, s'évertuaient à republier son Histoire socialiste de la Révolution française et à s'en inspirer, ni Mignet, ni Marx évidemment, ni d'autres plus récents, ne paraissent à lire Gueniffey, avoir énoncé à ce sujet quelques propositions dignes d'être évoquées. L'idée même d'observer les effets de transformation sociale qu'impliquerait l'action politique révolutionnaire à quoi se sont consacrés tant d'historiens, n'effleure guère notre savant essayiste en sorte que sa profession de foi gentiment réformiste de la page 50 ressortit plus à la parade verbale qu'à l'énonciation d'un principe, en vérité à le suivre, la politique ne peut devenir que l'accompagnement dans la sphère du pouvoir de ce que dicte la nature des choses et l'imperturbable compacité des héritages: l'apparent réformisme de la profession de foi se révèle n'être qu'une nouvelle figure du conservatisme et c'est Burke et nul autre que nous lisons en filigrane dans l'essai de Patrice Gueniffey. La probité aurait exigé que le maitre à penser soit évoqué sans esquive et que soit pleinement rendu à Carl Schmitt (La dictature..., tr.fr., 2000, ouvrage cité dans sa traduction italienne à la page 367 de la bibliographie de l'essai), ce qui en vérité lui est très visiblement dû.

4 Malgré cette réserve de fond qui a trait à la démarche idéologique et théorétique de Gueniffey, loin de nous est l'idée de mésestimer, au nom de l'orientation qui en fonde la démarche, l'intérêt ou la subtilité de nombre d'analyses, brillantes et souvent éloquentes de son livre. Le chapitre «Les ressorts de la Terreur» observe avec finesse et circonspection la mise en mouvement des répressions révolutionnaires de masse moins bien les insurrections -, regarde les "extrêmités» (p.260) auxquelles elles ont atteint, notamment en Vendée, les bricolages meurtriers sans cesse renaissants à la base ou du côté des exécutants et leur officialisation ambiguë et tardive, quoique ce soit précisément à travers ces formes incertaines que s'est établi un dispositif qui a conduit en l'an II à la déroute des forces contre-révolutionnaires et à l'inversion d'une situation politico-militaire majoritairement -Gueniffey n'en disconvient pas- défavorable dans l'été de 1793 et peut-être avant (cf. le chapitre V «Les circonstances et la guerre» que suit le chapitre «Les débuts de la Terreur [1791-1792]»). Tout aussi bien, la partie du chapitre consacrée au «legs de l'absolutisme» (p. 57) sait développer avec éloquence le contenu de la critique libérale, souvent reprise dans le cours du livre, qui, de Staël à Quinet et jusqu'à Furet, voit dans le despotisme révolutionnaire un produit adultérin de l'absolutisme monarchique fondé sur le principe «d'une extériorité du pouvoir par rapport à la société» (p.60), principe que les révolutionnaires ont à leur tour revendiqué contre «l'indépendance équivoque de l'individu» au profit des «droits de la cité» (Barère, cité p.l89) dont «l'État qui incarne la collectivité dans sa continuité» deviendra avec la Terreur, un innommable Moloch. Soit. Cependant la reprise d'une idée devenue courante ne lui donne pas pour autant le label de la vérité incontestable; $a$ fortiori quand sont évacuées sans la moindre justification, les analyses relatives aux effets cumulatifs de rupture que portaient en elles l'histoire du jansénisme et l'action des jansénistes depuis le milieu du xvire siècle, comme nous les a donnés à voir dans sa thèse, Catherine Maire. 
5 Relevons à l'opposé deux moments bien incertains dans la démonstration de Gueniffey. S'agissant du recours aux «circonstances» dans l'émergence et le renforcement de la Terreur, l'auteur qui se place dans le sillage de Furet, conteste l'hypothèse d'une corrélation positive entre accentuation des mesures de Terreur sous couvert de Salut public et aggravation de l'affrontement entre Révolution et Contre-Révolution (cf.chapitres X «Prairial» et XI «Idéologie et politique»). Il y voit l'effet d'une logique internaliste, principalement dominée en bout de période et s'agissant de Robespierre principalement, par une simple stratégie du coup d'État permanent (p.332). Observant la situation initiale et voulant récuser la démarche à la fois prosopographique et sociologique de Timothy Tackett (Par la volonté du peuple..., 1996, tr. fr. 1997), Gueniffey s'appuie - bien abusivement selon moi - sur les fines analyses biographiques de Edna Lemay pour combattre l'idée que les hommes de la Constituante ont changé, se sont transformés dans le temps même où leur action et la modification quelquefois de leur pensée théorique et politique, conduisaient à une transformation d'ensemble de la réalité sociale et des rapports de forces (cf. pp. 103-109). Mais ne craignant pas la contradiction, voire la cacophonie, l'auteur nous avait antérieurement exposé que 1793-1794 renoue avec «la logique du pouvoir constituant où l'idée de pouvoirs extraordinaires trouvait aisément sa place» (p.164) - l'observation du travail des divers Comités des recherches vient à l'appui de l'affirmation qui est exacte - tandis que, avant 1792, la «situation était différente» en raison de l'existence de la Constitution de 1791 qui marquait la limite entre les différents pouvoirs. On ne voit pas ici quelle logique interne pourrait expliquer de tels hiatus et de tels errements conceptuels, sauf à incriminer une situation nouvelle, de nouveaux enjeux, une configuration en somme surdéterminante, qui, d'un coup fait basculer la prétendue réfutation de la théorie mathiéziste des circonstances, du côté de ces constructions intellectuelles abstraites qu'aucune source ne vient étayer et qui vient comme justification rhétorique de points de vue préétablis.

6 Sur un autre plan encore, on concédera volontiers à l'auteur d'un essai qu'il n'a pas à s'encombrer de ces démonstrations d'ordre statistique dont l'historien ne saurait se passer et dont Gueniffey connait les vertus roboratives comme il l'a abondamment montré dans son précédent ouvrage relatif aux élections. Il est vrai que le comptage macabre des victimes est un exercice accablant dont il est cependant difficile de s'affranchir si l'on a le souci d'être exact. Gueniffey y consacre donc quelques pages fatiguées à la suite de l'intertitre: «Un bilan macabre» (p. 234). Car à moins de prendre en bloc tous les décès provoqués par mort violente en les rapportant au passif de «la» Révolution qui devient alors une sorte de manifestation d'un mal métaphysique, force est bien de tenter de mesurer le nombre des décès et des disparitions pour l'un et l'autre côté, l'autre côté étant ici celui des «bleus», des Constitutionnels, des élus et gardes nationaux, des protestants, des requis des départements, etc. Nombre d'historiens s'y sont récemment consacrés, souvent avec prudence, mais il ne reste pas grand chose de cette prudence dans le discours de Gueniffey: ainsi, pouquoi avoir cité en note «l'estimation haute» de J.-C. Martin tout en esquivant le devoir de suivre sa démonstration (n.15, p.234)? Pourquoi ne se fonder que sur les seules évaluations de Jacques Hussenet dans Recherches vendéennes, ou sur celles de Jacques Dupâquier qui participent du mythe du génocide franco-français, en évacuant d'un trait de plume ou par la mise sous le boisseau toutes les autres propositions? Et pas un mot ni une simple note de surcroît pour évoquer les recherches pionnières en ce domaine de François Lebrun, celles de Claude Petitfrère ou celles de Claudy Valin: peut-on ici faire silence 
sur de telles occultations dans un domaine aussi sensible? Il ne reste presque rien de la démarche prudente des historiens cités dans le livre de Gueniffey, lequel se réfère aussi bien au début de son livre aux évaluations fantasmatiques d'après Thermidor qu'à des données reçues et remises en circuit sans la moindre évaluation critique. Imaginons que la même désinvolture ait été manifestée par un quelconque historien, à propos des massacres de juin 1848 ou de la Terreur versaillaise de 1871-1873 (dont Gueniffey nous dit d'ailleurs que «la dictature exercée par le gouvernement révolutionnaire au nom de la Convention préfigure bien plutôt celle qu'exerceront le général Cavaignac [...] et Thiers [...]» [p.271] - ce qui est un comble! -) ou qu'on puisse énoncer quelque chose de comparable pour évoquer la Terreur patriotique anticollaborationniste de septembre à décembre 1944, laquelle succédait à la terreur milicienne et nazie des mois précédents, ou à propos des répressions coloniales, approuvées par gouvernements et Parlement et accompagnées de la torture, que n'entendrions-nous pas dire de la légéreté de l'essayiste qui s'en rendrait coupable? Mais quand il s'agit de la Révolution, pour beaucoup de ses contempteurs, l'à-peu-près et l'oui-dire paraissent suffisants...

7 À-peu-près et ouï-dire dont on trouve d'autres manifestations comme cette affirmation assez surprenante (p.273) selon laquelle si les révolutions de 1830 et 1848 n'ont pas abouti à la Terreur, c'est qu'on eut la sagesse de ne révoquer personne: outre que c'est faire peu de cas des travaux qui montrent comment 1830 comme 1848 ont modifié en profondeur la nature des enjeux sociaux et le jeu des forces politiques, on s'étonnera de voir ainsi sous-estimée la grande valse du personnel politico-administratif auquel on se livra après juillet 1830 sur fond de répression prolongée et renouvelée peu après contre les républicains. Quant à faire, peut-être pouvait-on aussi se demander si la mémoire de la radicalité révolutionnaire de 1793 n'était pas la cause première des compromis «en avant» qui s'imposèrent postérieurement: trop engelsien ou «marxiste» assurément! Enfin, dans les pages écrites à propos de la tradition historiographique qui voit dans la filiation du jacobinisme au républicanisme, un «legs» de la Révolution, mais sous la forme d'un «jacobinisme séparé de la Terreur» (p.203), Gueniffey nous la baille belle! Pour sa démonstration, il croit pouvoir s'appuyer sur le témoignage de l'ancien conventionnel Paganel, qualifié par lui de girondin et qui écrivait en $1806 \ldots$ Or, ce Paganel n'a jamais été girondin mais le type même du conventionnel de la Plaine. À tout prendre mieux valait faire appel au témoignage d'un vrai sympathisant de la Gironde, en l'occurrence, le conventionnel Bailleul, mais celui-ci en l'anV et de nouveau en 1817 en réponse à Germaine de Staël, n'hésita pas à justifier la Terreur dont il fut en partie victime, en raison des circonstances... comme quoi rien dans la réalité n'a la candide simplicité du discours guenifféen.

On lit, page 267: «À partir de l'automne de 1791, en s'affranchissant de toutes relations aux circonstances, la Terreur se développe dans le cadre des luttes pour la conquête d'un pouvoir sans base légale et en vue d'une révolution privée de tout objectif identifiable»: quels en sont les ressorts? La passion ou le délire de protagonistes médiocres et débridés, portés à l'exercice du pouvoir, la furieuse domination de savonaroles, privés, à la différence de leur prédécesseur, de toute foi et savoir (N.B.: l'allusion à Savonarole se trouve page 331). La théorie de Gueniffey s'épuise dans cet aveu final d'impuissance. Mais tout cela pour ça, comme on dirait dans un film de Lelouch, ne nous mène pas très loin d'un point de vue heuristique... mais très loin au contraire si l'objectif réellement poursuivi était d'exorciser le mal révolutionnaire toujours potentiellement renaissant, stigmatiser en somme à travers la dénonciation explicite du «jacobinisme», «la réalité atroce de toute révolution»(p.226): fin mot 
assurément d'un essai qu'on pourra à juste titre tenir pour un bricolage hâtif et daté, mais qui sera cité dans toutes les bibliographies, on peut s'en convaincre aisément, moins pour la rigueur démonstrative de son propos, que pour la pertinence idéologique de son dessein. 